\title{
The SPES project at the INFN- Laboratori Nazionali di Legnaro
}

G. Prete ${ }^{\mathrm{a}}$, A. Andrighetto, M.Manzolaro, S.Corradetti, D.Scarpa, M.Rossignoli, A.Monetti, M.Lollo, M.Calderolla, J.Vasquez, D.Zafiropoulos, L.Sarchiapone, D.Benini, P.Favaron, M.Rigato, R.Pegoraro, D.Maniero, L.Calabretta ${ }^{1}$, M.Comunian, M.Maggiore, A.Lombardi, L.Piazza, A.M.Porcellato, C.Roncolato, G.Bisoffi, A.Pisent, A.Galatà, M.Giacchini, G.Bassato, S.Canella, F.Gramegna, J.Valiente, J.Bermudez, P.F. Mastinu, J.Esposito, J.Wyss, S.Zanella.

INFN, Laboratori Nazionali di Legnaro, I-35020, Legnaro, Italy

${ }^{1}$ INFN, Laboratori Nazionali del Sud, I-95123, Catania, Italy

\begin{abstract}
The SPES Radioactive Ion Beam (RIB) facility at INFN-LNL is in the construction phase. It is based on the ISOL method with an UCx Direct Target able to sustain a power of $10 \mathrm{~kW}$. The primary proton beam is delivered by a high current Cyclotron accelerator, with energy $35-70 \mathrm{MeV}$ and a beam current of $0.2-0.5 \mathrm{~mA}$. Neutron-rich radioactive ions will be produced by proton induced Uranium fission in the UCx target at an expected fission rate in the order of $10^{13}$ fissions per second. The exotic isotopes will be re-accelerated by the ALPI superconducting LINAC at energies of $10 \mathrm{~A}$ $\mathrm{MeV}$ and higher, for masses in the region $\mathrm{A}=130 \mathrm{amu}$ at expected rate on the secondary target of $10^{7}-10^{9}$ pps. The SPES project has the aim to provide high intensity and highquality beams of neutron-rich nuclei as well as to develop an interdisciplinary research center based on the cyclotron proton beam.
\end{abstract}

\section{Introduction}

SPES is the acronym for "Selective Production of Exotic Species". The aim of the SPES project is to provide high intensity and high-quality beams of neutron-rich nuclei to perform forefront research in nuclear structure, reaction dynamics and interdisciplinary fields like medical, biological and material sciences. SPES is a second generation ISOL radioactive ion beam facility. It represents an intermediate step toward the future generation European ISOL facility EURISOL [1]. The SPES project is part of the INFN Road Map for the Nuclear Physics; it is supported by the Italian national laboratories LNL (Legnaro) and LNS (Catania). It is based on the ISOL method with an UCx Direct Target able to sustain a power of $10 \mathrm{~kW}$. The primary proton beam is delivered by a Cyclotron accelerator with an energy of more then $40 \mathrm{MeV}$ and a beam current of $200 \mu \mathrm{A}$. Neutron-rich radioactive ions will be produced by Uranium fission at an expected fission rate in the target of the order of $10^{13}$ fissions per second. The exotic isotopes will be re-accelerated by the ALPI superconducting LINAC at energies of $10 \mathrm{~A} \mathrm{MeV}$ and higher, for masses in the region of $\mathrm{A}=130 \mathrm{amu}$, with an expected rate on the secondary target of $10^{8} \mathrm{pps}$.

\section{SPES and the European context}

The European road map for nuclear physics with ISOL facilities goes through the development of second generation ISOL facilities pointing to EURISOL, the future European ISOL facility.

Actually three ISOL facilities are under construction in Europe [2]: HIE_ISOLDE, SPIRAL2 and SPES with the goal to offer to the European and international Nuclear Physics community up-to-date

\footnotetext{
${ }^{\mathrm{a}}$ Corresponding author: prete@lnl.infn.it
} 
neutron rich exotic beams and to improve the ISOL technology toward EURISOL. Expected performances for the new facilities are:

- to reach, and possibly overcome, $10^{13}$ fission per second in the production target optimizing the extraction efficiency (the neutron rich elements are mainly produced by Uranium fission induced by light ions or neutrons),

- to reaccelerate the exotic beams at energies of $10 \mathrm{~A} \mathrm{MeV}$ and over to address nuclear physics questions through a wide range of reaction mechanisms,

- to improve the selection capabilities of the beam handling systems producing more pure exotic beams.

At European level a strong collaboration is going on coordinated by Nupecc and funded by the ENSAR project that is part of the Seventh Framework Programme of the European Community. All the major nuclear physics European institutions are involved in order to carry out research at the forefront of fundamental nuclear science.

To define the physics and the users for SPES at a first call at end 2010, 24 letters of intent were presented by international collaborations focusing on transfer reactions, coulomb excitation, isospin effects and fusion-evaporation reactions with radioactive beams.

Up-to-date detectors are proposed for experiments, reshaping actual LNL detectors, like the PRISMA [3] spectrometer, exploiting new set-up as the GALILEO [4] gamma array under construction or performing campaign of measurements with complex detectors under construction among European collaborations (AGATA [5], NEDA [6], PARIS [7] and FAZIA [8]).

The most requested beam is the ${ }^{132} \mathrm{Sn}$ with the aim to study the shell evolution around the double magic numbers $\mathrm{Z}=50, \mathrm{~N}=82$

A number of dedicated mini-workshop are organized to define the First Day Experiments according to scientific priorities, instrumentation development and radioactive beam preparation.

\section{The SPES physics}

To understand the properties of a nucleus, apart from establishing the interaction between its components, it is necessary to determine the arrangement of the nucleons, i.e. the structure. Presently our knowledge about the structure of nuclei is mostly limited to nuclei close to the valley of stability or nuclei with a deficiency of neutrons. Only recently the availability of beams of unstable ions has given access to unexplored regions of the nuclear chart, especially on the neutron-rich side.

Starting from a nucleus on the stability line and adding successively neutrons, one observes that the binding energy of the last neutron decreases steadily until it vanishes and the nucleus decays by neutron emission. The position in the nuclear chart where this happens defines the neutron drip line. It lies much farther away from the valley of stability than the corresponding drip line associated with protons, owing the absence of electrical repulsion between neutrons. The location of the neutron drip line is largely unknown as experimental data are available only for nuclei with mass up to around 30 . The interest in the study of nuclei with large neutron excess is not only focused on the location of the drip line but also on the investigation of the density dependence of the effective interaction between the nucleons for exotic N/Z ratios. In fact, changes of the nuclear density and size in nuclei with increasing N/Z ratios are expected to lead to different nuclear symmetries and new excitation modes. While in the case of some very light nuclei a halo structure has been identified, for heavier nuclei the formation of a neutron skin has been predicted.

The evolution of nuclear properties towards the neutron drip line depends on how the shell structure changes as a function of neutron excess. This evolution has consequences on the ground state properties of the nuclei and on the single-particle and collective excitations. In particular, studies of neutron-rich nuclei beyond the doubly magic ${ }^{132} \mathrm{Sn}$ are of key importance to investigate the singleparticle structure above the $\mathrm{N}=82$ shell closure and find out how the effective interaction between valence nucleons behaves far from stability.

New modes of collective motion are also expected in connection with the formation of a neutron skin, namely oscillation of the skin against the core, similar to the soft dipole mode already identified 
in the case of very light halo nuclei. Presently, neither the thickness nor the detailed properties of the neutron skin of exotic nuclei are known. This information is needed to enable a quantitative description of compact systems like neutron stars, where exotic nuclei forming a Coulomb lattice are immersed in a sea of free neutrons, a system which is expected to display the properties of both finite and infinite (nuclear matter) objects. At the energy of SPES, it will be possible to address important questions related to the study of neutron-rich matter such as nuclear forces, level density, viscosity, barrier, neutron pairing and collective modes.

With the high intensity beams delivered by SPES, a challenging and broader range of studies in nuclear spectroscopy and reaction mechanism will be performed. Interesting areas where new data will be collected are those in the very neutron rich regions, where shell evolution is an issue. Effects of how the pairing interaction is modified in the nuclear medium will receive significant inputs by measurements of multi-nucleon transfer reactions to specific nuclear states. Effects of rotational damping in the decay of high energy levels, for instance the dynamical dipole emission, will be studied by changing the N/Z of projectile and target. Neutron rich nuclei will be used to investigate the tunneling process in sub-barrier fusion reactions in presence of very positive Q-values, an issue interesting also for astrophysics.

\section{Description of the SPES project}

SPES is a new mid-term ISOL facility dedicated to the production of neutron-rich beams. It is an INFN project involving the two national laboratories, LNL and LNS and other INFN sites in Italy. The ISOL (Isotope Separation On Line) [9] technique for radioactive beam production is based on a driver accelerator which induces nuclear reactions inside a thick target. The reaction products are extracted from the target by thermal process due to the high temperature of the target -ion source system (exceeding $2000^{\circ} \mathrm{C}$ ). After reaching the $1+$ source, strongly connected to the ISOL target, the products are ionized and extracted at $40 \mathrm{kV}$ producing a low energy radioactive beam ionized $1+$. After an isotopic selection and a further ionization to $n+$ the exotic beam is injected into a reaccelerator. In order to produce neutron-rich isotopes it is mandatory to perform fission reactions in Uranium or other actinide targets using protons, deuterons, neutrons or gammas.

The SPES choice is to use a proton beam to induce fission on a UCx target (Direct Target).

The driver is a $70 \mathrm{MeV}$ proton cyclotron with two exit ports for a total current of $750 \mu \mathrm{A}$ delivering beam on several targets. Two production ISOL targets are planned to be installed and operated alternatively.

As the cyclotron can supply two beams at the same time, a second independent facility can be operated in parallel. Interest has been already shown by other communities. In particular, the high intensity proton beam could be used to produce innovative radioisotopes for nuclear medicine as well as neutron beams in a wide energy spectrum which, in turn, is interesting for measurements of neutron capture reactions of astrophysical interest and the production of quasi mono-energetic neutron beam and atmospheric-like neutron spectra for Soft Error studies on electronic devices and systems. The expected neutron production at SPES exceeds $10^{14} \mathrm{n} / \mathrm{s}$ with energy up to $70 \mathrm{MeV}$ and a peak between 0.1 and $10 \mathrm{MeV}$ according to target and moderator. It is possible to simulate the generation IV reactor spectra to study material cross-sections and activation. SPES participates in the Union for Compact Accelerator-based Neutron Sources UCANS [10].

The proton beam of SPES cyclotron is similar to the ARRONAX [11] one and it is suited for the production of new radioisotopes for medical applications. Taking advantage of the high energy and current, it opens the possibility to produce ${ }^{82} \mathrm{Sr},{ }^{67} \mathrm{Cu}$, and ${ }^{47} \mathrm{Sc}$ to be used in therapy and diagnostics. Specific area and beam lines are dedicated to these applications, a further building expansion to other applications is also possible. A pictorial sketch of the facility is shown in figure 1. The SPES ISOL facility will produce neutron-rich beams from Uranium fission with a design goal to reach a rate of $10^{13}$ fission/sec in the production target. This fission rate requires a proton beam of $40 \mathrm{MeV} 200 \mu \mathrm{A}$. 


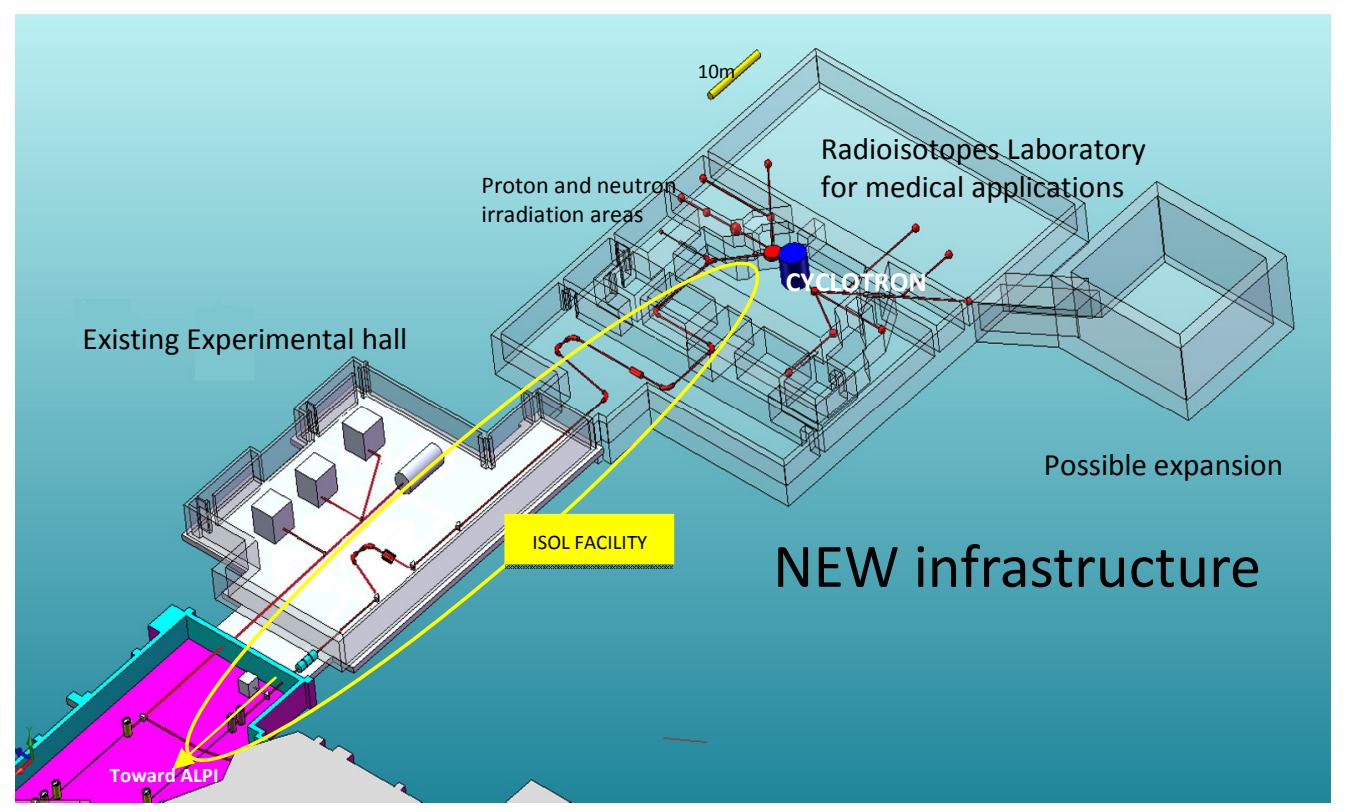

Figure 1. Pictorial sketch of the SPES facility. ALPI superconductive linac not shown on the left side

\subsection{The ISOL front-end}

The ISOL front-end is the system which couples the proton beam with the UCx target, the ion source and the first part of the transport line of the exotic beam. SPES adopted the ISOLDE design. The whole system was completed and is in operation at LNL for off-line tests.

\subsubsection{The ISOL target system}

The most critical element of the SPES ISOL facility is the Direct Target. The proposed target follows the basic design of the ISOLDE one but represents an innovation in term of capability to sustain the primary beam power. The design is carefully oriented to optimize the radiative cooling, taking advantage of the target system's high operating temperature, which is of the order of $2000^{\circ} \mathrm{C}$.

The SPES target is designed in order to maximize the release efficiency and the heat dissipation as well as to exploit devices (basically the ion sources) developed in other laboratories. The SPES target consists of multiple thin disks housed in a cylindrical graphite box. It is composed of $7 \mathrm{UC}_{\mathrm{x}}$ disks (diameter and thickness of 40 and $1.3 \mathrm{~mm}$, respectively), appropriately spaced in the axial direction in order to dissipate, by thermal radiation, the average power of $8 \mathrm{~kW}$ due to the proton beam which, passing through them and induces nuclear reactions. The graphite box is housed inside a tubular hollow tungsten ohmic heater. In fact, due to the intense heat exchange by radiation, the proton beam is not sufficient to reach the operating temperature. This allows for a better thermal control of the target operation.

An extensive simulation of the target behaviour for thermal and release properties is at the basis of the target-ion-source design. Experimental work to benchmark the simulations was carried out in collaboration with HRIBF [12], the Oak Ridge National Laboratory ISOL facility (USA). Partial results on the measured production and the expected reaccelerated SPES beam is shown in figure 2 . 


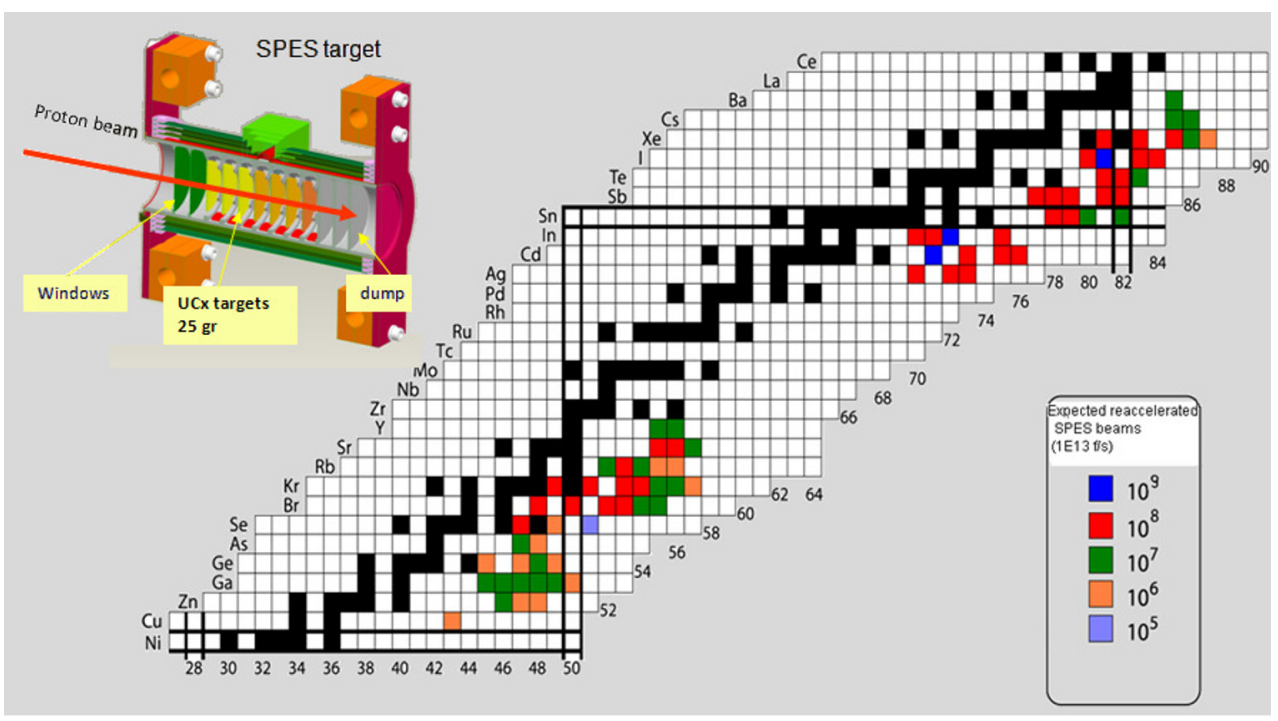

Figure 2. Expected beam on target. Experimental data scaled to: $0.2 \mathrm{~mA}$ proton current, $2-5 \%$ transport efficiency. ISOL target operated at $2000^{\circ} \mathrm{C}$, ionization and extraction with $1+$ Plasma Source.

\subsubsection{The ion-source system}

The interaction of the proton beam with the UCx target will produce fission fragments of neutron-rich isotopes that will be extracted by thermal motion and ionized at $1^{+}$charge state by a source directly connected with the production target. The hot-cavity ion source chosen for the SPES project was designed at CERN (ISOLDE) [5]. The source has the basic structure of the standard high temperature RIB ion sources employed for on-line operation. The ionizer cavity is a W tube ( $34 \mathrm{~mm}$ length, $3 \mathrm{~mm}$ inner diameter and $1 \mathrm{~mm}$ wall thickness) resistively heated to near $2000^{\circ} \mathrm{C}$. The isotopes produced in the target diffuse in the target material and after that will effuse through the transfer tube (its length is approximately $100 \mathrm{~mm}$ ) into the ionizer cavity where they undergo surface or laser ionization. The Surface ionization process can occur when an atom comes into contact with a hot metal surface. In the positive surface ionization, the transfer of a valence electron from the atom to the metal surface is energetically favorable for elements with an ionization potential lower than the work function of the metal. For alkalis and some rare earth elements, high ionization efficiencies can be achieved using the surface ionization technique. This 1+ source has good efficiency and selectivity for the elements as $\mathrm{Rb}, \mathrm{Cs}, \mathrm{Ba}$. For most part of the others elements, the laser resonant photo-ionization, using the same hot cavity cell, is a powerful method to achieve sufficiently selected exotic beams. This technique is under development implemented with the aim to produce beams as pure as possible (chemical selectivity) also for metal isotopes. To ionize elements with high ionization potential, as rare gasses the plasma source is needed. This source ionizes all the elements without any selectivity. At SPES both surface and plasma sources are developed and in operation at the off line test stand. The laser source is under development.

\subsection{Beam selection and transport}

The first mass selection is performed by a Wien Filter with 1/100 mass resolution, installed just after the first electrostatic quadrupole triplet inside the production bunker with the aim to confine the larger part of radioactivity inside the high shielded area. The transfer line toward ALPI is equipped with several beam handling systems to purify the beam: a Beam Cooler and a High Resolution Mass Separator (HRMS) with 1/20000 mass resolution will be installed inside the new building. A crucial task for the experiment with radioactive beams is not only the beam intensity but also the beam 
quality. Special efforts have been dedicated to design a mass spectrometer with an effective mass resolution of at least $1 / 20000$. Such design takes advantage of a Beam Cooler, to improve the beam emittance, and of a $260 \mathrm{keV} \mathrm{HV}$ platform on which the mass separator is mounted. The mass separator is a scaled-up version of the separator designed by Cary Davids for CARIBU [13], Argonne. In the SPES configuration the physics design gives a resolution on the order of 1/40000 constrained by an emittance of $3 \pi \mathrm{mm}$ mrad and energy spread of $1.3 \mathrm{eV}$. Such high selectivity results in an advantage also for the safety issue, reducing the problems of contaminations along the beam transport area, re-accelerator and final target location.

Before the injection in the ALPI Linac it is necessary to increase the charge state from $1+$ to $n+$ for an effective reacceleration. This is performed by means of a Charge Breeder. The SPES Charge Breeder is based on ECR method [14] and aims to produce ions with A/q less than 6 at A 130. The design and construction will be performed in the framework of the SPES-SPIRAL2 collaboration at LPCS (Grenoble, France) following an up-graded version of the Phoenix booster in operation at LPSC.

After the Charge Breeder a second mass separator with 1/1000 mass resolution, will be installed to clean the beam from the contaminants introduced by the Charge Breeder itself (CB-MS). The same basic configuration of HRMS is adopted. Finally an RFQ pre-accelerator will increase the beam energy to match the ALPI acceptance. The layout of the radioactive beam line is showed in figure 3 .

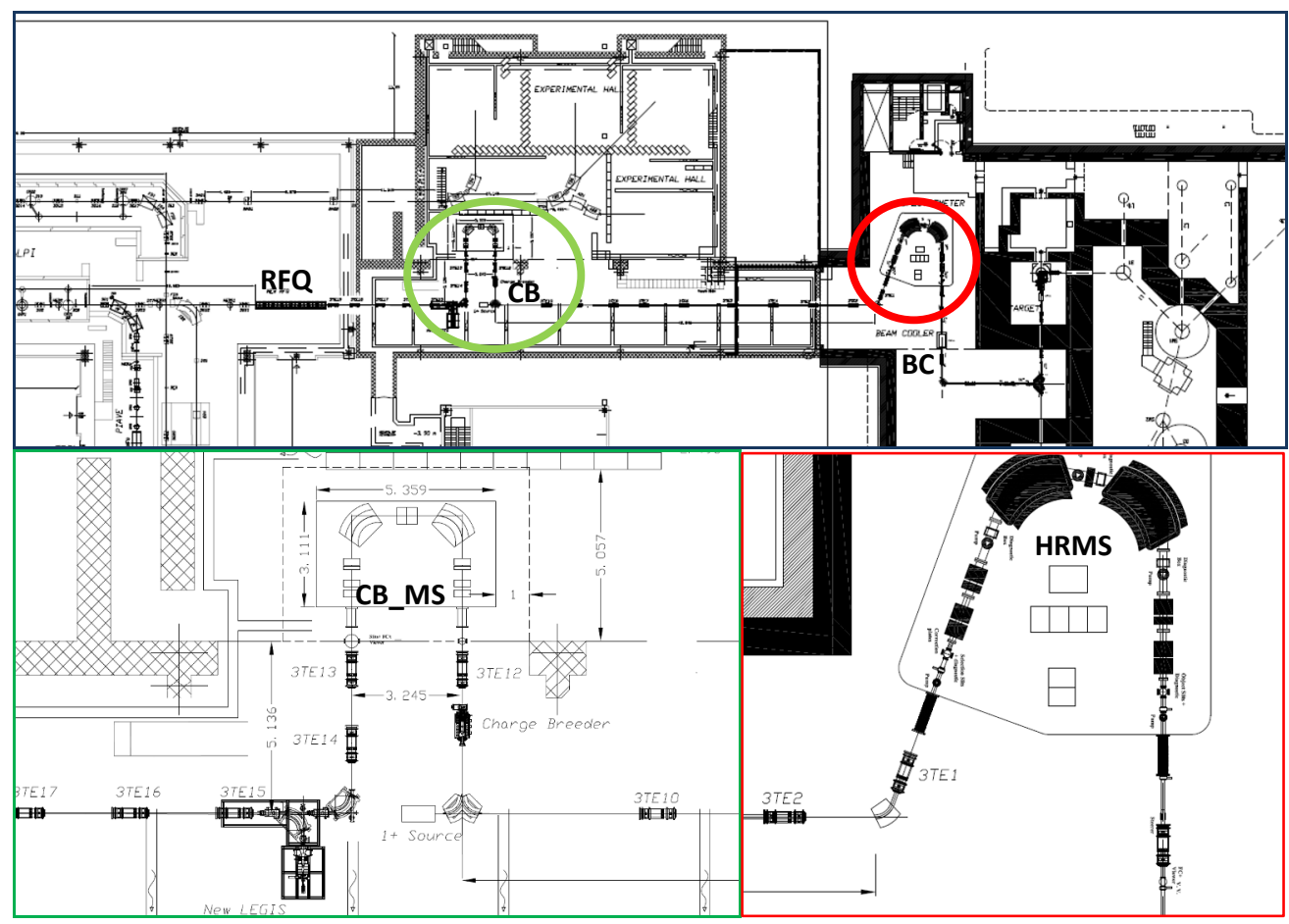

Figure 3. Layout of radioactive beam handling and transfer line. High resolution mass separator and the Charge Breeder mass separator are shown in the lower panels.

\subsection{Exotic beam reacceleration}

This SPES injector for ALPI includes a new RFQ (see Tab. 8.4) that will operate in a CW mode (100\% duty factor) at a resonant frequency of $80 \mathrm{MHz}$. This frequency is the same as that of the superconducting structures of the lowest energy section of ALPI. The injection energy of ions was set to $5.7 \mathrm{keV} / \mathrm{u}$. This choice is a compromise between the desire to reduce the ion energy to simplify the 
LEBT and the RFQ bunching section design and the need to increase the beam rigidity in the 1+ transport line to reduce space charge effects. The extraction energy of the RFQ was set to $727 \mathrm{keV} / \mathrm{u}$ to optimize the beam dynamics of the superconducting linac ALPI.

Table 1. Main parameters of the SPES pre-acceleration RFQ

\begin{tabular}{|l|l|}
\hline Parameter (units) & Value \\
\hline Operational mode & $\mathrm{CW}$ \\
\hline Frequency $(\mathrm{MHz})$ & 80. \\
\hline Injection Energy $(\mathrm{keV} / \mathrm{u})$ & $5.7(\beta=0.0035)$ \\
\hline Output Energy $(\mathrm{keV} / \mathrm{u})$ & $727(\beta=0.0395)$ \\
\hline Accelerated beam current $(\mu \mathrm{A})$ & 100 \\
\hline Charge states of accelerated ions $(\mathrm{Q} / \mathrm{A})$ & $7-3$ \\
\hline Transmission $(\%)$ & 95 \\
\hline Internal bunching section & Yes \\
\hline
\end{tabular}

ALPI is the superconducting heavy ion LINAC under operation at LNL since the early nineties. It is composed by three sections of quarter wave resonators (QWR) for a total of 80 cavities installed and operates routinely at an equivalent acceleration voltage of $50 \mathrm{MV}$. The LINAC is constructed in a bended configuration: it is composed by two branches connected by an achromatic and isochronous U-bend. It uses three different kinds of cavities: Low Beta, Medium Beta and High Beta cavities, according to the different velocity along the acceleration path. The ALPI linac has seen a continuous upgrade in the number and performance of its accelerating cavities, and consequently of the maximum achievable beam energy. In the last years the cavities of the medium energy QWR section were upgraded using a new $\mathrm{Nb}$ sputtered coating in substitution to the original $\mathrm{Pb}$ sputtered layer. A further upgrade program is on the way, to improve the accelerating fields of the present QWRs and adding more cavities in the Low Beta section. The final equivalent voltage will exceed $70 \mathrm{MV}$ so as to go well beyond the Coulomb barrier for nuclear reactions involving mid-A projectiles and heavy targets. In figure 3 the expected beam energies are reported.

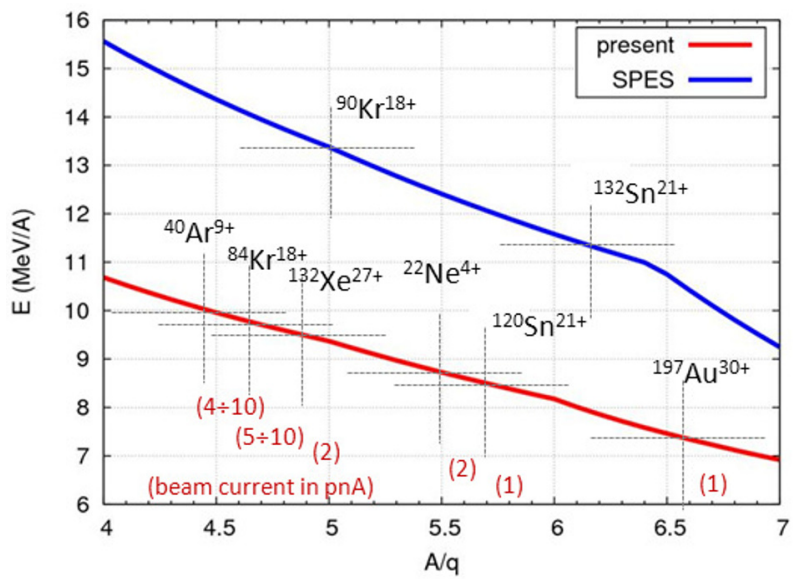

Figure 3. Beam energy performance of ALPI superconductive Linac

\section{Status of the SPES project}

SPES is under construction at LNL. The building is at the excavation phase and will be completed at end 2014. The cyclotron is under construction by BEST in Canada, the magnet is completed and the 
magnetic field was positively mapped. The cyclotron is expected to be transferred to LNL after the factory commissioning in the second quarter of 2014. The authorization to operate the cyclotron was already obtained at LNL allowing to accelerate the proton beam on non-fissile target at high power and testing the UCx target up to $5 \mu \mathrm{A}$ current. A further authorization request to run the $\mathrm{UCx}$ target at design power will be presented to the Italian authorities at the end of this year. The physics design of the radioactive ion beam transfer line $(1+$ and $n+)$ is completed and the reshaping of the ALPI beam line in the experimental hall is initiated to allow the installation of the Charge Breeder and the CB mass separator. The ISOL front-end is completed and under operation in the off-line laboratory to test ion sources, assembly and controls. The first reaccelerated exotic beam is expected in 2017 as shown in table 2.

Table 2. SPES project planning

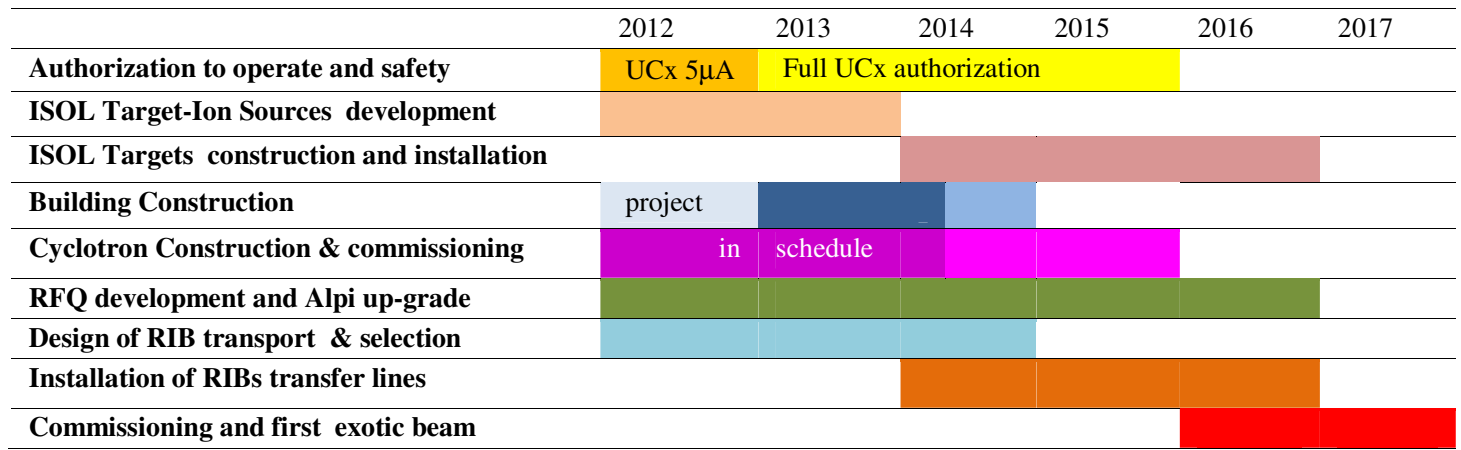

\section{References}

1. www.eurisol.org

2. European Science Foundation, Perspectives of Nuclear Physics in Europe, NuPECC Long Range Plan 2010, ISBN: 978-2-918428-29-9

3. A.M. Stefanini et. al, Nuclear Physics A 701 (2002) 217

4. Calin A.Ur, Journal of Physics 366 (2012) 012044

5. E. Farnea, F.Recchia, D.Bazzacco, Th.Kroll, Zs.Podolyak, B.Quintana, A.Gadea, The AGATA Collaboration, Nucl. Instr. and Meth. A 621, 331-343 (2010)

6. G.Jaworski et al. NIM A673 (2012) 64

7. A. Maj et al., "The PARIS Project", Acta Phys. Pol. B40 (2009)

8. http://fazia.in2p3.fr/Fazia/fazia.htm

9. M.Lindroos, CERN-AB-2004-086, (2004)

10. http://www.indiana.edu/ lens/UCANS/

11. Haddad F, Ferrer L, Guertin A, Carlier T, Michel N, Barbet J, Chatal JF, .Eur. J. Nucl. Med. Mol. Imaging. 35(7), 1377 (2008)

12. D. Scarpa, L. Biasetto, S. Corradetti, M. Manzolaro, A. Andrighetto, S.Carturan, G. Prete, P. Zanonato and D.W. Stracener Eur. Phys. J. A (2011) 47

13. Savard, G.; Pardo, R.; Baker, S.; Davids, C.; Levand, A.; Peterson, D.; Phillips, D.; Sun, T.; Vondrasek, R.; Zabransky, B.; Zinkann, GHyperfine Interactions, Vol. 199 Issue 1-3, (2011) 301

14. T. Lamy, J.-L. Bouly, J.-C. Curdy, P. Sole, P. Sortais, T. Thuillier, J.-L. Vieux-Rochaz, and D. Voulot ; Rev. Sci. Instrum. 75, 1624 (2004) 